\title{
Knowledge of developmental pharmacology and modeling approaches should be used to avoid useless trials in children
}

\author{
Saskia N. de Wildt • Catherijne A. J. Knibbe
}

Received: 31 March 2009/Accepted: 2 April 2009 /Published online: 25 April 2009

(C) The Author(s) 2009. This article is published with open access at Springerlink.com

In their recent paper, Tafuri et al. [1] argue that although a drug is used in an off-label manner in children, enough evidence may be available from existing studies to not warrant further studies. As researchers in pediatric clinical pharmacology and practicing clinicians, we strongly support this approach. The use of data from existing, often investigator-initiated, studies to determine the appropriateness of off-label use of drugs may greatly reduce the burden on children to participate in possibly unnecessary studies.

In their paper, these authors propose that the availability of empirical data from two pharmacokinetic (PK) and two efficacy studies in children is enough to waive additional studies. In this respect, we would like to emphasize the impressive physiological changes and, consequently, PK and pharmacodynamic (PD) changes that occur in children over the whole age continuum [2]. In order to account for these developmental changes, three aspects of drug trials in children should be taken into account when available studies are being assessed: the choice of the dose, the choice of the group (age and disease) and the choice of the endpoints to measure the effect of the drug. If these aspects are not adequately addressed in the trial (design), the researcher(s) may arrive at the wrong conclusion(s) and

\footnotetext{
S. N. de Wildt $(\triangle) \cdot$ C. A. J. Knibbe

Intensive Care Unit, Erasmus MC Sophia Children's Hospital, Dr Molewaterplein 60, Sk3140,

3015 GJ Rotterdam, The Netherlands

e-mail: s.dewildt@erasmusmc.nl

C. A. J. Knibbe

Clinical Pharmacy, St. Antonius Hospital,

Nieuwegein, The Netherlands

C. A. J. Knibbe

Leiden Amsterdam Center for Drug Research, Leiden University, Leiden, The Netherlands
}

subsequently impose on children a treatment that lacks efficacy or, even worse, expose them to the risk of drug toxicity.

An example of how the choice of the dose may lead to incorrect study conclusions is a randomized clinical trial of diphenhydramine in children reported in 2006 [3]. In this trial, where bodyweight-corrected adult doses of diphenhydramine were used in infants, no effect of the drug on sleep was observed in this population [3]. However, the sizecorrected clearance of the drug was found to be much higher in infants than in adults [4]. It may therefore be concluded that the doses used in the efficacy study were probably too low to reach drug concentrations associated with sleep in adults.

This example stresses the need to take into account agerelated differences in the dose-concentration relationship between children of different ages and adults when interpreting efficacy studies. In addition, treatment indications, disease processes, outcome of therapy and the concentration-effect relationship may also differ for children of different ages. Hence, the effect of age on all of these determinants of drug therapy outcome should be considered before data from children of one age group are used to waive the need for studies in another age group. As such, in this context, it is evident that the availability of data from two PK and two efficacy studies of a protein pump inhibitor (PPI) for the treatment of heartburn complaints in adolescents should not be used to waive studies on the efficacy of a PPI for respiratory symptoms related to gastroesophageal reflux disease in preterm infants. More specifically, most of the studies reported in the article of Tafuri et al. [1] were performed in children older than 1 or 2 years. However, it is known that the largest developmental changes in PK and PD are likely to occur in the age range younger than 1 year and that these 
are the most striking during the first weeks of life. These developmental changes necessitate that studies be performed in the youngest age groups as well before conclusions on the use in the entire group of children can be drawn. Additionally, the effect of the drug in children should be measured using validated age-appropriate endpoints, which may vary between the youngest children versus older children and adults.

We agree with Tafuri et al. [1] on the need to incorporate existing knowledge and would like to specify the potential role of population PK-PD modeling. This tool allows for the analysis of existing in vitro and in vivo data from children, which results in the design of dosing guidelines for children of different ages without the need for extensive clinical trials [5]. Additionally, this approach also allows for minimization of the need for additional studies in children because developmental changes in PK and PD can be characterized even when only sparse sampling is applied. In case data are lacking in specific age groups of for specific indications and additional studies are thus needed, the use of limited sampling strategies based on simulations using population PK-PD modeling will reduce the burden for individual patients [5].

Finally, it is important to address the question of whom should be responsible for assessing the appropriateness of the use of an off-label drug in children. Currently, this responsibility has been left with the treating physician, guided by clinical guidelines or information from drug formularies - if these are available at all. We believe that this responsibility should reside with the regulator(s). The regulator has not only access to published study data but also to unpublished data from the manufacturer. These existing data should be carefully evaluated by regulators with expertise in developmental pharmacology and appro- priate modeling techniques. The regulator can then decide, based on this evaluation, whether to waive or reduce the need for additional studies and grant a license for the appropriate age range and treatment indication-or not. While this would mean a substantial effort by the regulatory authorities, such a procedure would lead to major improvement in pharmacotherapy in children, from both an ethical and medical point of view.

Open Access This article is distributed under the terms of the Creative Commons Attribution Noncommercial License which permits any noncommercial use, distribution, and reproduction in any medium, provided the original author(s) and source are credited.

\section{References}

1. Tafuri G, Trotta F, Leufkens HG, Martini N, Sagliocca L, Traversa G (2009) Off-label use of medicines in children: can available evidence avoid useless paediatric trials? The case of proton pump inhibitors for the treatment of gastroesophageal reflux disease. Eur J Clin Pharmacol 65(2):209-216

2. Abdel-Rahman SM, Reed MD, Wells TG, Kearns GL (2007) Considerations in the rational design and conduct of phase I/II pediatric clinical trials: avoiding the problems and pitfalls. Clin Pharmacol Ther 81(4):483-494

3. Merenstein D, Diener-West M, Halbower AC, Krist A, Rubin HR (2006) The trial of infant response to diphenhydramine: the TIRED study-a randomized, controlled, patient-oriented trial. Arch Pediatr Adolesc Med 160(7):707-712

4. Simons KJ, Watson WT, Martin TJ, Chen XY, Simons FE (1990) Diphenhydramine: pharmacokinetics and pharmacodynamics in elderly adults, young adults, and children. J Clin Pharmacol 30 (7):665-671

5. Ince I, de Wildt SN, Tibboel D, Danhof M, Knibbe CA (2009) Tailor-made drug treatment for children Creation of an infrastructure for data-sharing and population PK-PD modeling. Drug Discov Today 14(5-6):316-320 